\title{
Fungal Enzymes for Bio-products from Sustainable and Waste Biomass
}

Vijai K. Gupta ${ }^{1 *}$, Christian P. Kubicek ${ }^{2}$, Jean-Guy Berrin ${ }^{3,4}$, David W. Wilson ${ }^{5}$, Marie Couturier $^{3,4}$, Alex Berlin ${ }^{6}$, Edivaldo X. F. Filho ${ }^{7}$ and Thaddeus Ezeji ${ }^{8}$

${ }^{1}$ Molecular Glycobiotechnology Group,

Discipline of Biochemistry, National University of Ireland Galway, Galway City, Ireland

${ }^{2}$ Biotechnology and Microbiology,

Institute of Chemical Engineering

TU Wien, Gumpendorferstrasse 1a/1/166.5, 1060 Wien, Austria

${ }^{3}$ INRA, UMR1163 Biodiversité et Biotechnologie Fongiques, 163 avenue de Luminy, 13288

Marseille, France

${ }^{4}$ Aix Marseille Université, UMR1163 Biodiversité et Biotechnologie Fongiques, 163 avenue de Luminy, 13288 Marseille, France ${ }^{5}$ Department of Molecular Biology and Genetics, Cornell University, Ithaca, New York 14853, USA ${ }^{6}$ Novozymes, Inc., 1445 Drew Ave, Davis CA 95618, USA ${ }^{7}$ Laboratory of Enzymology, Department of Cell Biology, University of Brasilia, block K floor, Asa Norte , 70910-900 - Brasilia, DF-Brazil

${ }^{8}$ Biotechnology and Fermentation Group, Department of Animal Sciences, The Ohio State University \& OARDC, 1680 Madison Avenue, Room 305 Gerlaugh Hall Wooster Ohio 44691, USA

*Corresponding author: vijaifzd@gmail.com (V.K. Gupta) 


\begin{abstract}
Lignocellulose, as the most abundant renewable carbon source on earth, is the logical candidate to replace fossil carbon as the major biofuel raw material. Yet, the technologies needed to convert lignocellulose into soluble products that can then be utilized by the chemical or fuel industries still face a number of challenges. Enzymatic hydrolysis is of major importance there. Herein, we review the progress made in fungal enzyme technology over the past few years with major emphasis on (i) the enzymes needed for the conversion of polysaccharides (cellulose and hemicellulose) into soluble products, (ii) the potential uses of lignin degradation products and (iii) the current progress and bottlenecks for the use of the soluble lignocellulose derivatives in emerging biorefineries.
\end{abstract}

Keywords: fungal enzymes, renewable biomass, CAZymes, xylan-degrading enzymes, lignin modifications, enzymatic hydrolysis

\title{
Fungal degradation of lignocellulosic biomass
}

Lignocellulose, as the most abundant renewable carbon source on earth, is an obvious candidate for replacement of fossil carbon. It is also attractive because its use avoids the direct fuel-versus-food competition that is debated when corn and cane feedstocks are used for production of fuels and biochemicals in biorefineries. To this end, technologies are needed to convert lignocellulose into soluble products, such as, mono or disaccharides and lignin-degradation products. The past few years have seen increased bioprospecting activities to isolate and develop novel fungal enzyme mixtures, which can be tailored to efficiently degrade recalcitrant lignocellulose into monomeric sugars with mild or no thermochemical pretreatment [1-2]. Complete deconstruction of lignocellulose to fermentable sugars by enzymes requires the concerted activities of ligninases (manganese peroxidase, lignin peroxidase, versatile peroxidase, and laccase), endoglucanases and exoglucanases (acting on 
reducing and non-reducing ends of cellulose), with or without a carbohydrate-binding module $(\mathrm{CBM})$, as well as various hemicellulases. The enzyme mix should likewise contain accessory enzymes and proteins, such as the lytic polysaccharide monooxygenases (LPMOs) and swollenins [3-7]. The presence or addition of enzymes that depolymerize pectin and cleave side-chain substituents can enhance, in some cases, the rate of lignocellulose hydrolysis (Figures 1 and 2) [8,].

This review collates the current knowledge on the fungal enzymes needed for the biotechnological conversion of lignocellulosic biomass into soluble products that can be used by the biorefinery industry.

\section{Lignocellulose determinants for its hydrolysis}

Lignocellulose is a heterogeneous, multi-component, polymeric material that forms a complex structure with a three dimensional network, which typically consists of cellulose (40-50\%), hemicellulose (25-30\%), and lignin (15-20\%). Fungi are potent degraders of cellulose, which they use as carbon source. Lignocellulolytic fungi are widely distributed throughout the fungal kingdom, from the simplest and primitive Chytridiomycetes to the most advanced Basidiomycetes. Fungi predominantly and selectively degrade lignin, cellulose, and hemicellulose, (e.g. white rot fungi), degrade polysaccharides and modify lignin (e.g. brown rot fungi), or degrade lignin and polysaccharides simultaneously (e.g. soft rot fungi) [9-10]. These fungi produce repertoires of enzymes (Figure 1) either individually or as a consortium to degrade and metabolize recalcitrant lignocellulose.

Yet evolutionary adaptation has provided plants with barriers that are aimed at inhibiting the penetration and colonization of the plant cell wall by microorganisms, with help of secreted cell-wall degrading enzymes, so they can generate enough carbon and other nutrients and secure their survival in nature. One hypothesis has attributed recalcitrance of lignocellulose to the arrangement of its monomeric units [11]. Only those microorganisms 
that can decrypt the underlying code of the plant cell wall assembly will be able to attack and efficiently hydrolyze its constituent polymers to their oligomeric and monomeric building blocks.

The limited accessibility of cellulose and hemicellulose in plant cell walls to microorganisms has also been explained by their interaction and/or chemical association with lignin and the relative ratio of its monomer constituents. Lignin is composed of three monolignol monomers, which can be methoxylated to various degrees: $p$-coumaryl alcohol, coniferyl alcohol, and sinapyl alcohol. These lignols are incorporated into lignin in the form of the phenylpropanoids p-hydroxyphenyl (H), guaiacyl (G), and syringyl (S) units, respectively. Recently, it has been shown that transgenic poplar lines that are extremely rich in syringyl lignin exhibited a drastically improved resistance to degradation by all decay fungi [12]. Finally, the distribution and sequence of the monosaccharide units, the various glycosidic linkages, and the vicinity and location of each branch point within lignocellulose have a significant impact on its three dimensional microstructure and thus increase lignocellulose resistance to hydration and decrease the accessibility of the cellulose before and after pretreatment [11-12]. There are other factors that affect digestibility of cellulose and hemicellulose by hydrolytic enyzmes, but they present no bottlenecks to degradation by fungal enzymes, and will thus not be covered here.

\section{Enzymatic degradation of cellulose}

\section{The cellulolytic system}

Fungi, as the dominant players in recycling of cellulose in nature, are well-adapted for the degradation of cellulosic biomass: for this purpose they produce a high number of enzymes of broad variety and with complementary activities. The cellulolytic model system to date is that of the ascomycete fungus Trichoderma reesei, because it is used in many biotechnological industries and it serves as a progenitor for the generation of a multitude of 
mutants capable of secreting in submerged fermentations cellulolytic enzymes with yields exceeding $100 \mathrm{~g}$ enzyme protein/L whole fermentation broth. However, several other fungi other than Trichoderma reesei, such as Penicillium verruculosum or Chrysosporium lucknowense, have also been reported to be excellent cellulase and hemicellulase producers [13]. Shrestha et al. [14] isolated 106 fungi from decaying leaves of Miscanthus and sugar cane and detected several that secreted a superior enzyme mixture than those already under study. Enzymes involved in polysaccharide deconstruction are grouped in the Carbohydrate Active Enzyme (CAZy) classification based on comparison of their amino acid sequences, three-dimensional structures and catalytic mechanisms [15-17]. Cellulases belong to the Glycoside Hydrolase (GH) family. More recently, the Auxiliary Activity (AA) families of enzymes has been added to CAZy [18], which contain the recently discovered LPMOs. Furthermore, the database contains non-catalytic modules (Carbohydrate Binding Modules, CBMs), which are involved in substrate targeting and are appended to many GH enzymes [19]. The most prevalent fungal CBMs binding specifically critalline cellulose are from the family 1 (CBM1). Family 6 CBMs bind predominately to amorphous cellulose and are rare, while family $63 \mathrm{CBMs}$ are also rare. There are many other fungal CBMs that bind to other polysaccharides. They often increase the overall catalytic activity of CAZymes, especially on crystalline substrates.

Historically, a system of three complementary enzymatic activities has been proposed to be responsible for cellulose degradation: endoglucanases, cellobiohydrolases and $\beta$ glucosidases [20]. These enzymes are able to hydrolyze the $\beta-1,4$ covalent bonds that connect the glucose units in cellulose chains. Endoglucanases (EG, endo-1,4 $\beta$ D-glucanases, EC 3.2.1.4) randomly cleave $\beta-1,4$ bonds in amorphous areas of cellulose and generate new reducing and non-reducing ends (Figure 2). These are classified into several CAZy families, namely GH5, GH7, GH12, and, GH45, and share a common structure that includes a large 
cleft containing the catalytic amino acids. Cellobiohydrolases (CBHs, cellulose 1,4- $\beta$ cellobiosidases, EC 3.2.1.91) are processive enzymes that release cellobiose (i.e. two units of glucose linked by a $\beta-1,4$ bond) from either reducing ( $\mathrm{GH} 7 \mathrm{CBHs}$ ) or non-reducing ends (GH6 CBHs) of cellulose fragments produced by endoglucanases. CBHs contain tunnelbearing structures, which allow the enzyme to slide along the cellulose chain to the next cleavage site as the product is released. $\beta$-glucosidases (GH1 and GH3) hydrolyse cellobiose or cello-oligosaccharides into glucose. They are characterized by a pocket-containing topology for optimal detection of the non-reducing extremity and cleave off a single sugar unit. $\beta$-glucosidases are non-processive enzymes, since the substrate has to be released after each cleavage event to allow the new glucose molecule to exit the pocket. Both exoglucanases and $\beta$-glucosidases are strongly inhibited by their reaction productscellobiose and glucose, respectively.

\section{Fungal Auxiliary Proteins involved in Cellulose Degradation}

Most cellulolytic microorganisms secrete a large set of proteins when grown on cellulosic substrates, among which are enzymes with auxiliary activities (AA in CAZy) that act synergistically with cellulases. Aerobic fungi contain multiple AA9 LPMOs in their genomes that are mostly active on cellulose. LPMOs perform a copper-mediated oxidative cleavage of cellulose at the $\mathrm{C} 1$ and/or $\mathrm{C} 4$ position of the cellulose chain [18, 21-24]. Also, AA9 enzymes acting on xylan [25] and xyloglucan have been described [26-27]. During enzymatic attack, LPMOs cycle between $\mathrm{Cu}$ (I) and $\mathrm{Cu}$ (II) to activate molecular oxygen, whereby a copper-oxyl radical abstracts a hydrogen and then hydroxylates the substrate via an oxygen-rebound mechanism [28]. The initial oxygen species is proposed to be a super oxide [29]. They require a reducing agent, which in vitro can be satisfied by glutathione and ascorbate. In fungi, cellobiose dehydrogenase is considered as the main redox partner of LPMOs being secreted under cellulolytic conditions [30-33]. Most recently Westereng et al. 
[27] showed that the electrons can also be donated by long-range electron transfer involving high and low molecular weight lignins present in plant cell walls. Because of their synergistic action with cellulases, AA9 LPMOs are now part of commercial cellulase preparations [34], and their significantly reduces enzyme loading.

Expansins are non-catalytic proteins that can play a role in cellulose degradation although their mechanism is still unclear [35]. Disruption of cellulose fibers or reduction of substrate viscosity was observed in several instances [28] but until recently no hydrolytic activity was reported. The swollenin $\operatorname{TrSwo} 1$ is an expansin-like protein from $T$. reesei, that is capable of disrupting cellulose fibers [36], and which is also found in some other fungi [6]. Andberg et al. [5] have been able to detect degradation products from $\beta$-glucans and revealed that the mode of action of this protein appears to be similar to that of both endo- and exoglucanases, with cellobiose being the major degradation product.

\section{Kinetics of cellulose degradation and synergism}

Although the combination of cellulolytic enzymes from different $\mathrm{GH}$ families is theoretically enough to carry out complete conversion of cellulose into monomers, in practice complex kinetics, cellulose crystallinity, product inhibition, protein degradation, among other factors, lead to limited degradation efficiency. The kinetics of enzymatic cellulose degradation is complex and many models have been proposed to understand the activity of cellulases on cellulose over the course of degradation. Bansal et al. [37] have summarized the sequence of steps involved in cellulose degradation, which include adsorption of endoglucanase and cellobiohydrolase onto their substrate, release of the cellulase molecule from the cellulose chain, release of cellobiose and cellodextrin from the cellulose molecule, and hydrolysis of cellobiose and cellodextrin into glucose by $\beta$-glucosidases. Many factors affect kinetics, decreasing the rate of reaction over time. For example, substrate crystallinity is a major factor behind the decreasing hydrolysis reaction rate, since amorphous regions are 
hydrolysed first followed by the hydrolysis of more crystalline regions, resulting in a substrate richer in highly recalcitrant crystalline regions $[4,38]$.

Cellulolytic enzymes with different substrate specificities exhibit synergistic action (indicated by a higher hydrolytic activity than would be predicted the sum of the hydrolytic activities of the individual enzyme components) in the hydrolysis of cellulosic fibers. Thereby, endo-exo and exo-exo synergisms have been distinguished [4, 8]. For instance, endo-exo synergy occurs between $T$. reesei endoglucanase $\operatorname{TrCel5A}$ (EGII) and its cellobiohydrolase partner $\operatorname{Tr}$ Cel7A (CBHI), such that $\operatorname{Tr}$ Cel7A (CBHI) hydrolyzes from the reducing end of cellulose chains while $\operatorname{Tr}$ Cel6A (CBHII) hydrolyzes from the non-reducing ends. Interestingly, during a real-time representation of crystalline cellulose degradation by T. reesei CBHs utilizing high-speed atomic force microscopy [37], $\operatorname{Tr}$ Cel7A molecules were seen to slide unidirectionally along the crystalline cellulose surface. However, at one point the $\operatorname{TrCel7A}$ molecules displayed collective stops analogous to a traffic jam. Addition of $\operatorname{Tr}$ Cel6A resulted in a remarkable increase in the extent of mobile enzyme molecules on the surface. Cellulolytic glycoside hydrolases also exhibit strong synergism with their oxidative counterparts, the LPMOs. . The reason for their synergism appears to be the fact that AA9 LPMOs act at the surface of cellulose fibers and cellulases hydrolyse chains of cellulose [19, 21].

\section{Enzymatic degradation of hemicelluloses}

The enzymatic break down of hemicellulose requires a diverse set of enzymes acting on both terminal and/or internal glycosidic linkages. They include non-arabinose- and arabinose-liberating endo-xylanases, endo-xylanases (GH families 10, 11, 30, 43, and 51), $\beta$ xylosidases ( $\mathrm{GH}$ families 30, 39, 43, and 51), xyloglucanases ( $\mathrm{GH}$ families 12 and 74), acetyl xylan esterases (CE families 1-6) and ferulic esterases (CE family 1), $\beta$-mannanases (GH families 5, 26, and 134), $\alpha$-galactosidases ( $\mathrm{GH}$ families 27 and 36), $\alpha$ - 
arabinofuranosidases ( $\mathrm{GH}$ families 2, 3, 43, 51, 54 and 62) and $\alpha$-glucuronidases (GH families 67 and 115). Figure 3 shows an overview of hemicellulase action on a lignocellulosic substrate. The coordinated distribution of these enzymes on lignocellulosic substrates requires an enzymatic network or interactome of enzymes, acting in dynamic and cooperative ways in time and space [39-41]. These interactions require enzyme systems displaying hydrolytic abilities at macroscopic (spatial organization of plant cell wall structure) and molecular (breakdown at polymeric and oligomeric levels) levels.

Dixon [40] stressed that the lack of understanding of interaction and synergism of these enzymes has limited the design of plants that have reduced recalcitrance. Understanding the mechanism of enzymatic hydrolysis of hemicelluloses [42] is complicated by the complexity of the hemicellulase system and the structural and morphological heterogeneity of the substrate. Zhang et al. [43] developed a mechanistic model for synchronous enzymatic hydrolysis of cellulose and hemicelluloses. The model considers the substrate morphologies and their coupling with morphology-depended substrate hydrolysis kinetics, and was developed in order to present a replication of real-world hydrolysis and to allow the prediction of the enzymatic accessibility and conversion level for both hemicellulose and cellulose within substrates..In this work, two concepts were proposed to describe the morphology of hemicellulose-cellulosic substrates: SAV, which is the smallest void that can be attacked by enzyme molecules, and SAC, which is the minimal volume by external and internal surfaces exposed to enzyme-accessible hydrated interior voids of the solid substrate material, taking into consideration the intertwining between hemicellulose and cellulose chains. Furthermore, plant cell wall hemicelluloses have motifs that are recalcitrant to hydrolysis which are named pointrons, whereas motifs that are available to enzyme attack are named pexons [11]. This concept can be useful to address fundamental questions in the enzymatic hydrolysis of the plant cell wall, particularly for hemicellulases, some of which act 
on a range of structurally similar substrates whereas others catalyze alternative reactions on a range of substrates. Hemicellulases from different families can be used synergistically to create an enzymatic arsenal for the complete breakdown of hemicellulose. Thus, comprehensive studies of synergism can lead to the identification of tailor-made enzyme systems, optimization of their relative ratios and the rational design of enzymatic mixtures with better efficiencies, providing a basis for the formulation of active enzyme mixtures for efficient lignocellulose saccharification and modification [44-47]. So far, studies on the synergy of hemicellulases have identified only some of the interactions that can take place. In addition, the divergent evolution of hemicellulases in different fungi led to modulations in the structure of the active sites, the presence of multifunctional domains, and fusions with different carbohydrate binding domains [48], which may strongly influence the synergism shown by these enzymes. Thus, the scenario of hemicellulose breakdown in the plant cell wall includes the recruitment of hemicellulases with different mechanisms of action [40] and enzymes are selected based on hemicellulose complexity [42].

Proteomic, genomic and transcriptomic analyses are important tools that were used to reveal the functional diversity of yet uncharacterized hemicellulases in hemicellulose breakdown, which may not be deciphered from analysis of single mRNAs and proteins [49]. Indeed, the predicted proteomes of 103 representative fungi belonging to Ascomycota, Basidiomycota, Chytridiomycota, and Zygomycota phyla showed [50] that glycoside hydrolases (GH) and ), polysaccharide lyases, carbohydrate esterases (CE) are the most common CAZymes in all fungi analysed. For example, members of families CE1 and GH10, which present the common activities of carboxylesterase and endo-1,4- $\beta$-xylanase, respectively and have an incredible diversity in substrate specificity, were found to be present in all fungi examined. Computational and manual analyses were used in order to update and 
refine annotation of the CAZyme gene content of filamentous fungi and to study the functional diversification of filamentous fungal CAZyme genes [51].

\section{Industrial uses of lignocellulose hydrolysis products}

Most of the plant cell wall hydrolysates are used for biofuel production [52]. Since biofuels are not always cost competitive with fossil fuels [53], glucose is increasingly used for the production of platform chemicals (i.e. chemicals that serve as a convenient starting material for the synthesis of various products) [54]. New information from genome sequencing and advances in sophisticated techniques for metabolic engineering have allowed the development of robust and efficient strains capable of converting lignocellulose hydrolysates to platform chemicals. Table 1 lists the microorganisms and products that are currently under intensive research.

For the economic use of lignocellulosic feedstocks, all sugars in the lignocellulose hydrolysate, including D-xylose and L-arabinose, must be converted to the target products. Unfortunately, the two major biofuels producers, S. cerevisiae and Zymomonas mobilis, are unable to metabolize xylose and arabinose because they lack some of the genes and enzymes necessary for the uptake and metabolism of these two sugars. In addition, engineering xylose metabolism into recombinant yeast causes perturbation of the redox balance and xylitol, one of the sweetener platform chemicals, is toxic to Zymomonas [55]. Therefore, two alternative attempts have been devoted to render S. cerevisiae capable of xylose utilization: the introduction of xylose reductase and xylitol dehydrogenase (termed "oxidoreductasebased pathway"), and the introduction of bacterial xylose isomerase (termed "isomerase pathway"). Due to the cofactor imbalance associated with the oxidoreductase pathway, the xylose isomerase-based pathway has been suggested as the most promising strategy [56]. Recently, S. cerevisiae SXA-R2P-E, which harbors the xylose isomerase-based pathway, exhibited excellent xylose fermentation performance with an improved xylose consumption 
rate [57]. Co-fermentation in mixed sugars $(7 \%, w / v, D$-glucose and $4 \%$, w/v, D-xylose), lead to the formation of $50 \mathrm{~g} / \mathrm{L}$ of ethanol, equalling $0.43 \mathrm{~g}$ ethanol/g sugars [58].

Another major problem with the use of lignocellulose hydrolysates as a fermentation substrate is the presence of lignocellulose-derived microbial inhibitory compounds [LDMICs]. These LDMICs inhibit cell growth, and, thus, reduce product formation [59]. LDMICs can be derived from the plant biomass itself (e.g. acetic acid, ferulic acid), or, more frequently, be generated as side products of the pretreatment process (e.g. degradation products of xylose and glucose such as furfural and hydroxylmethyl furfural, and ligninderived phenolics). Potential strategies to alleviate the inhibitory effects of LDMICs to enhance lignocellulose hydrolysates utilization include metabolic engineering based on the knowledge of potential tolerance mechanisms and evolutionary engineering. Indeed, furfural toxicity in $E$. coli causes cofactor imbalance during sulfate assimilation [60-62], and can thus be mitigated by deleting a gene encoding a high affinity NADH-dependent-oxidoreductase or replacing it with a transhydrogenase. Tolerance towards ionic liquids, used for the pretreatment of lignocellulose, can be increased by engineering $E$. coli strains with an efflux pump [63].

Simultaneous use of all lignocellulose-derived sugars would enable the fast and complete conversion of lignocellulose to products [64]. However, whereas S. cerevisiae contains a multitude of hexose transporters that mediate the uptake of glucose [65], it lacks specific pentose transporters. One approach consisting of the expression of specific xylose transporters from other organisms has met little success, mainly because of inefficient targeting to the plasma membrane and degradation [56]. However, S. cerevisiae contains at least eight hexose transporters (Hxt) that mediate D-glucose uptake [66], and which can also take up D-xylose albeit with lower affinity [67]. Shin et al. [68] used evolutionary engineering of a D-xylose-fermenting $S$. cerevisiae strain lacking the major transporter 
HXT1-7 and GAL2 genes to obtain a mutant strain that expresses the normally cryptic HXT11 gene. A further mutation at N366(M/T) of Hxt11 reversed the transporter specificity for D-glucose into D-xylose while maintaining high D-xylose transport rates. The Hxt11 mutant enabled the efficient co-fermentation of xylose and glucose at industrially relevant sugar concentrations when expressed in a strain lacking the HXT1-7 and GAL2 genes [66, $68]$.

Alternatively, some bacteria have already been engineered to use cello-oligosaccharides directly, enabling the simultaneous use of cellobiose and xylose [69-70]. Extending this idea, engineering strains for the direct use of cellodextrins and xylo-oligosaccharides has an additional benefit of allowing the direct use of only partially depolymerized lignocellulosic biomass feedstock, thus reducing the number of exogenous enzymes needed for lignocellulose conversion to fuels and chemicals. Of particular interest is B-glucosidase, which is often the limiting enzyme in the cellulase preparations from T. reesei. Proof of principle of this concept has been demonstrated by engineering bacterial oligosaccharide transporters into E. coli [71-72], and by engineering Neurospora cellodextrin transporters into S. cerevisiae [73-74] and Y. lipolytica [75] for which the dependence on B-glucosidase for lignocellulose utilization was drastically reduced or eliminated.

\section{Enzymatic and microbial transformation of industrial lignins}

Industrial lignins, so-called, "technical lignins", are by-products of industrial processes that use lignocellulose biomass as the starting raw material. Examples of technical lignins are: "kraft lignin", a lignin by-product from the most commonly found in the Pulp and Paper industry process - the kraft process, or, "hydrolysis lignin" a by-product of the second generation biomass biorefineries [76]. As a result of the biomass physico-chemical processing, the chemical structure of technical lignins differs dramatically from that of native 
lignin that occurs naturally in plants. Technical lignins tend to be chemically more complex and structurally heterogenous and therefore present a new challenge for biodegradation. Fungal lignin transformations, both of native and industrial lignins, have focused traditionally on the lignin biodegrading action of white rot fungi, such as, Phanerochaete chrysosporium [77-79]. However, recent studies have shown that there are other fungal species with proven capacity to degrade industrial lignins efficiently. For instance, Bjerkandera adusta can degrade technical lignins from a variety of biomass pulping processes, such as, kraft, organosolv, and soda pulping [80]. Another fungus, Penicillium thomii, is capable of cleaving $\beta-\mathrm{O}-4$ bonds in kraft lignin. In addition, four fungal strains, Cryptococcus podzolicus, Sphaerulina polyspora, Phoma herbarum, and Davidiella tassiana have been reported to grow on lignosulphonates as sole carbon source [81].

Lignin-degrading microorganisms secrete ligninases, a myriad of complex enzymes such as, peroxidases and laccases, which work in concert to degrade lignin to its phenolic building blocks. These enzymes target lignin carbon-carbon and carbon-oxygen bonds, including $\beta-\mathrm{O}-4, \beta-5, \beta-\beta, 5-5,5-\mathrm{O}-4$, and, $\beta-1$ linkages, which hold together the complex three dimensional lignin structure [76] Four types of ligninases are a class of copperenzymes, called laccases, and three types of peroxidases, lignin peroxidases, versatile peroxidases, and manganese peroxidases. Laccases are not strong oxidants but they synergize with naturally occurring chemical mediators, such as acetosyringone, syringaldehyde, or $p$ coumaric acid, to degrade lignin. This synergy is currently viewed as one of the potential "breakthrough" applications for lignin valorization (see below; [82]). Unlike laccases, lignin peroxidases show very high redox potential, which allows for efficient oxidation of model phenolic compounds. However, depolymerization of lignin by lignin peroxidases is inefficient due to steric limitations and their tendency to repolymerize depolymerized lignin [83]. Manganese peroxidases, which are commonly found in white-rot fungi, are strong 
oxidants of phenolic compounds (especially their chelated forms), and are the only heme peroxidases capable of oxidizing $\mathrm{Mn}^{2+}$ to $\mathrm{Mn}^{3+}$. Furthermore, versatile peroxidases, are characterized by broad specificity for aromatic substrates, which makes them useful in various applications, in particular, in bioremediation of recalcitrant pollutants - are now of major industrial interest [84].

The composition of depolymerized lignin varies quite significantly depending on its processingand this is illustrated by their differences in physoc-chemical properties. For instance, native lignins, is insoluble in water and organic solvents, but certain industrial lignins, the lignosulfonates are water-soluble. The structure of industrial lignins is often condensed (specially in the case of processes, where strong acids are used for biomass fractionation), making it more chemically inert, and the presence of sulfur in kraft lignins can inhibit chemical catalysts (for review see [85]).

The development of fungal enzymes for industrial lignin depolymerisation or chemical functionalization has been hampered by the difficulties of growing white rot fungi on an industrial scale or - alternatively - by the expression of lignin-degrading enzymes in other fungi [83, 86]. Bacterial biocatalysts for lignin depolymerisation have therefore gained increasing interest, and this subject has been recently reviewed [87]. As an alternative, pathway engineering could potentially be used to engineer the lignin degradation pathways of a microbe that possesses the metabolic capability to break down lignin, in order to produce useful chemicals. As an example, vanillic acid is an intermediate in bacterial lignin breakdown, and deletion of the vanillin dehydrogenase gene in $R$. jostii RHA1 leads to the accumulation of vanillin, a high value chemical used in the food/flavour industry, together with 4-hydroxybenzaldehyde and ferulic acid [88]. Rhodococcus opacus has been shown to accumulate triglyceride lipids when growing on organosolv lignin extracts [89]. The bacterium Pseudomonas putida is a well-known degrader of aromatic substances that also 
possesses the ability to break down lignin. Linger et al. [90] have demonstrated that $P$. putida converts lignin into polyhydroxyalkanoate biopolyesters. Blockage of the protocatechuate cleavage pathway, and re-routing via the catechol cleavage pathway by metabolic engineering has rendered $P$. putida to accumulate cis, cis-muconic acid, can then be converted via catalytic hydrogenation into the valuable platform chemical adipic acid [91].

Despite its conversion into platform chemicals, polymeric lignins as well as depolymerised lignin breakdown products may have alternative uses, such as the production of phenolformaldehyde adhesives, phenolic resins and polyurethane foams [85]. There is also the possibility to convert lignin into carbon fibre, porous carbon or lignin nanocontainers, if highly purified lignin preparations can be obtained, however, there are challenges in generating high quality lignin precursors of carbon fibre, due the difficulty of generating highly purified lignin fractions from heterogeonous lignin preparations [92-94].

\section{Concluding remarks}

The synergy between fungal enzymes has been recognized as an effective strategy for bioproduct development from biomass. In this review, we have discussed the advantages and shortcomings of the use of fungal enzyme cocktails for efficient bioconversion of heterogeneous lignocellulose and waste biomass feedstock to value-added products such as fuels and chemicals.

To economically and sustainably produce biofuels and chemicals from renewable resources, we conclude that integration of the fungal omic technologies, including genomic, transcriptomic, proteomic, and interactomic, for efficient enzymatic breakdown of cell walls of plants will be strategic for a better understanding of individual and interactive roles of glycosyl hydrolase, carbohydrate esterase, ligninase and fungal auxiliary proteins in lignocellulose degradation. 


\section{Acknowledgement}

E. X.F.Filho acknowledges the support from the Brazilian National Council for Scientific and Technological Development (CNPq); and T. Ezeji would like to acknowledge funds from the Ohio Agricultural Research and Development Center (OARDC) and the USDA-NIFA Hatch project $\mathrm{OHO} 01333$.

\section{References}

1. Berrin, J.G. et al. (2012) Exploring the natural fungal biodiversity of tropical and temperate forests toward improvement of biomass conversion. Appl. Environ. Microbiol. 78, 6483-90. DOI: 10.1128/AEM.01651-12.

2. Couturier, M. et al. (2012) Post-genomic analyses of fungal lignocellulosic biomass degradation reveal the unexpected potential of the plant pathogen Ustilago maydis. BMC Genom. 2, 13-57. DOI: 10.1186/1471-2164-13-57.

3. Harris, P.V. et al. (2010) Stimulation of lignocellulosic biomass hydrolysis by proteins of glycoside hydrolase family 61, structure and function of a large, enigmatic family. Biochemistry 49, 3305-3316.

4. Hu, J., et al. (2014) Substrate factors that influence the synergistic interaction of AA9 and cellulases during the enzymatic hydrolysis of biomass. Energy Environ. Sci.7, $2308-2315$

5. Andberg, M. et al. (2015) Swollenin from Trichoderma reesei exhibits hydrolytic activity against cellulosic substrates with features of both endoglucanases and cellobiohydrolases. Bioresour Technol.181, 105-13.

6. Kang, K. et al. (2013) Characterization of a novel swollenin from Penicillium oxalicum inacilitating enzymatic saccharification of cellulose. BMC Biotechnol. 13,42, DOI: $10.1186 / 1472-6750-13-42$ 
7. Gourlay, K. et al. (2013) Swollenin aids in the amorphogenesis step during the enzymatic hydrolysis of pretreated biomass. Bioresour Technol. 142, 498-503. DOI: 10.1016/j.biortech.2013.05.053.

8. Yang, B. et al. (2011) Enzymatic hydrolysis of cellulosic biomass. Biofuels 2, 421450.

9. Maitan-Alfenas, G.P. et al. (2015) Enzymatic hydrolysis of lignocellulosic biomass, converting food waste in valuable products. Curr Opin Food Scie. 1, 44-49.

10. Sigoillot, J.C. et al. (2012) Fungal Strategies for Lignin Degradation. Adv. Bot. Res. 61,263-308, DOI:10.1016/B978-0-12-416023-1.00008-2

11. Eveline, Q.P.T. and Buckeridge, M.S. (2015) Do plant cell walls have a code? Plant Science 241, 286-294

12. Skyba, O. et al. (2013) Syringyl-rich lignin renders poplars more resistant to degradation by wood decay fungi. Appl. Environ. Microbiol. 79, 2560-2571

13. Gusakov, A.V. Alternatives to Trichoderma reesei in biofuel production. Trends Biotechnol. 29, 419-425.

14. Shrestha, P. et al. (2015) Fungi isolated from Miscanthus and sugarcane: biomass conversion, fungal enzymes, and hydrolysis of plant cell wall polymers. Biotechnol Biofuels 5, 8:38.

15. Martinez, D. et al. (2008) Genome sequencing and analysis of the biomassdegrading fungus Trichoderma reesei (syn. Hypocrea jecorina). Nat. Biotechnol. 26, $553-560$

16. www.cazy.org

17. www.cazypedia.org 
18. Lombard, V. et al. (2014) The Carbohydrate-active enzymes database (CAZy) in 2013. Nucleic Acids Res 42, D490-D495.

19. Levasseur, A. et al. (2013) Expansion of the enzymatic repertoire of the CAZy database to integrate auxiliary redox enzymes. Biotechnol Biofuels. 6, 41, DOI: $10.1186 / 1754-6834-6-41$

20. Várnai, A. et al. (2014) Carbohydrate-binding modules of fungal cellulases, occurrence in nature, function, and relevance in industrial biomass conversion. Adv. Appl. Microbiol. 88, 103-165.

21. Payne, C.M. et al. (2015) Fungal cellulases. Chem. Rev. 115, 1308-1448

22. Frandsen, K.E. et al. (2016) The molecular basis of polysaccharide cleavage by lytic polysaccharide monooxygenases. Nat Chem Biol. 12, 298-303.

23. Johansen, K.S. et al. (2016) Discovery and industrial applications of lytic polysaccharide mono-oxygenases. Biochem Soc Trans. 44, 143-149.

24. Corrêa, T.L. et al. (2016) AA9 and AA10, from enigmatic to essential enzymes. Appl Microbiol Biotechnol. 100, 9-16

25. Frommhagen, M. et al. (2015) Discovery of the combined oxidative cleavage of plant xylan and cellulose by a new fungal polysaccharide monooxygenase. Biotechnol. Biofuels. 2015; 8:101

26. Hemsworth, G. R. et al. (2014) Discovery of a new family of lytic polysaccharide mono-oxygenases. Nat. Chem. Biol. 10, 122-126.

27. Westereng, B. et al. (2015) Enzymatic cellulose oxidation is linked to lignin by longrange electron transfer. Sci Rep. 21, 5:18561, DOI: 10.1038/srep18561.

28. Kim, S. et al. (2014) Quantum mechanical calculations suggest that lytic polysaccharide monooxygenases use a copper-oxyl, oxygen-rebound mechanism. Proc Natl Acad Sci USA 111, 149-154, DOI: 10.1073/pnas.1316609111 
29. Kjaergaard, C. H. et al. Spectroscopic and computational insight into the activation of $\mathrm{O}-2$ by the mononuclear $\mathrm{Cu}$ center in polysaccharide monooxygenases. Proc Natl Acad Sci USA 111, 8797-8802, DOI: 10.1073/pnas.1408115111

30. Bennati-Granier, C. et al. (2015) Substrate specificity and region-selectivity of fungal AA9 lytic polysaccharide monooxygenases secreted by Podospora anserina. Biotechnol. Biofuels 8, 90.

31. Navarro D. et al. (2014) Fast solubilization of recalcitrant cellulosic biomass by the basidiomycete fungus Laetisaria arvalis involves successive secretion of oxidative and hydrolytic enzymes. Biotechnol Biofuels. 7:143.

32. Langston, J.A. et al. (2011) Oxidoreductive cellulose depolymerization by the enzymes cellobiose dehydrogenase and glycoside hydrolase 61. Appl Environ Microbiol.77, 7007-15.

33. Agger, J. W. et al. (2014) Discovery of LPMO activity on hemicelluloses shows the importance of oxidative processes in plant cell wall degradation. Proc. Natl Acad. Sci. USA $111,6287-6292$.

34. Morgenstern, I. et al. (2014) Fungal cellulose degradation by oxidative enzymes: from dysfunctional GH61 family to powerful lytic polysaccharide monooxygenase family. Brief. Funct. Genomics 13, 471-481

35. Liu, X. et al. (2015) Research advances in expansins and expansion-like proteins involved in lignocellulose degradation. Biotechnol. Lett. 37, 1541-51

36. Saloheimo, M. et al. (2002) Swollenin, a Trichoderma reesei protein with sequence similarity to the plant expansins, exhibits disruption activity on cellulosic materials. Eur. J. Biochem. 269, 4202-4211

37. Bansal, P. et al. (2009) Modeling cellulase kinetics on lignocellulosic substrates. Biotechnol. Adv. 27, 833-848 
38. Igarashi, K. et al. (2011) Traffic jams reduce hydrolytic efficiency of cellulase on cellulose surface. Science 333, 1279-82

39. Siqueira, F. G. and Filho, E. X. F. (2010) Plant Cell Wall as a Substrate for the Production of Enzymes with Industrial Applications. Mini-Rev. Org. Chem. 7, 54-60.

40. Dixon, R. A. (2013) Microbiology: Break down the walls. Nature 493, 36-37.

41. Monclaro, A. V. et al. (2014) The Role of $\alpha$-Glucuronidases in the Deconstruction of Plant Cell Wall Structure. In Fungal Enzymes (1st edn) (Polizeli, M.L.T.M. and Rai, M., eds), pp. 280-293, CRC Press.

42. Álvarez et al. (2016) Enzymatic Hydrolysis of biomass from wood. Microb. Technol. $9,149-156$

43. Zhang, Y. et al. (2014) On a Novel Mechanistic Model for Simultaneous Enzymatic Hydrolysis of Cellulose and Hemicellulose Considering Morphology. Biotechnol. Bioeng. 111, 1767-1781

44. Harris, P.V. et al. (2014) New enzyme insights drive advances in commercial ethanol production. Curr. Opin. Chem. Biol. 19, 162-170

45. Goldbeck, R. et al. (2014) Development of hemicellulolytic enzyme mixtures for plant biomass deconstruction on target biotechnological applications. Appl. Microbiol. Biotechnol. 98, 8513-8525

46. Laothanachareon, T. et al. (2015) Synergistic action of recombinant accessory hemicellulolytic and pectinolytic enzymes to Trichoderma reesei cellulase on rice straw degradation. Biores. Technol. 198, 682-690

47. Bhattacharya, A. and Pletschke, B. I. (2015) Strategic optimization of xylanasemannanase combi-CLEAs for synergistic and efficient hydrolysis of complex lignocellulosic substrates. J. Mol. Catal. B, Enzym 115, 140-150 
48. Van Dyk, J. S and Pletschke, B. I. (2012) A review of lignocellulose bioconversion using enzymatic hydrolysis and synergistic cooperation between enzymes-factors affecting enzymes, conversion and synergy. Biotechnol. Adv. 30, 1458-1480

49. Haider, S. and Pal, R. (2013) Integrated Analysis of Transcriptomic and Proteomic Data. Curr. Genomics 14, 91-110

50. Zhao, Z. et al. (2013) Comparative analysis of fungal genomes reveals different plant cell wall degrading capacity in fungi. BMC Genomics 14,274, DOI: 10.1186/14712164-14-274

51. Häkkinen, M. et al. (2012) Re-annotation of the CAZy genes of Trichoderma reesei and transcription in the presence of lignocellulosic substrates. Microb. Cell Fact. 11, 134; DOI: 10.1186/1475-2859-11-134

52. Kircher, M. (2015) Sustainability of biofuels and renewable chemicals production from biomass. Curr. Opin. Chem. Biol. 29, 26-31

53. Kircher, M. (2014) The emerging bioeconomy, industrial drivers, global impact, and international strategies. Ind. Biotechnol. 10, 11-18

54. Chen, R. and Dou, J. (2015) Biofuels and bio-based chemicals from lignocellulose, metabolic engineering strategies in strain development. Biotechnol. Lett. 38, 213-221.

55. Agrawal, M. et al. (2011) Adaptation yields a highly efficient xylose-fermenting Zymomonas mobilis strain. Biotechnol. Bioeng. 108,777-805

56. Moysés, D.N. et al. (2016) Xylose Fermentation by Saccharomyces cerevisiae: Challenges and Prospects. Int J Mol Sci. 2016 Feb 5;17(3). pii: E207.

57. Lee, S.M. et al. (2014) Alper. Systematic and evolutionary engineering of a xylose isomerase-based pathway in Saccharomyces cerevisiae for efficient conversion yields. Biotechnol. Biofuels, 7, 122, DOI: 10.1186/s13068-014-0122-x 
58. Ko, J.K. et al. (2016) Ethanol production from lignocellulosic hydrolysates using engineered Saccharomyces cerevisiae harboring xylose isomerase-based pathway. Bioresour Technol. 209, 290-6.

59. Cray, J. A. et al. (2015) Chaotropicity, a key factor in product tolerance of biofuelproducing microorganisms. Curr. Opin. Biotechnol. 33, 228-259.

60. Miller, E.N. et al. (2010) Genetic changes that increase 5-hydroxymethyl furfural resistance in ethanol-producing Escherichia coli LY180. Biotechnol. Lett. 32, 661667

61. Wang, X. et al. (2013a) Engineering furfural tolerance in Escherichia coli improves the fermentation of lignocellulosic sugars into renewable chemicals. Proc. Natl. Acad. Sci .USA 110, 4021-4026

62. Wang, X. et al. (2013b) Metabolomic analysis reveals key metabolites related to the rapid adaptation of Saccharomyce cerevisiae to multiple inhibitors of furfural, acetic acid, and phenol. OMICS 17, 150-159

63. Frederix, M. et al. (2014) Development of a native Escherichia coli induction system for ionic liquid tolerance. PLoS One 9, e101115, DOI:10.1371/journal.pone.0101115

64. Chen, R. (2015) A paradigm shift in biomass technology from complete to partial cellulose hydrolysis, lessons learned from nature. Bioengineered. 6, 69-72

65. Young, E.M. et al. (2012) A molecular transporter engineering approach to improving xylose catabolism in Saccharomyces cerevisiae. Metab Eng. 14, 401-411.

66. Sedlak, M. and Ho, N.W.Y. (2004) Characterization of the effectiveness of hexose transporters for transporting xylose during glucose and xylose co-fermentation by a recombinant Saccharomyces yeast. Yeast Chichester Engl. 21, 671-684. 
67. Saloheimo, A. et al. (2007) Xylose transport studies with xylose-utilizing Saccharomyces cerevisiae strains expressing heterologous and homologous permeases. Appl Microbiol Biotechnol. 74, 1041-1052

68. Shin, H.Y. et al. (2015) An engineered cryptic Hxt11 sugar transporter facilitates glucose-xylose co-consumption in Saccharomyces cerevisiae. Biotechnol Biofuels. 8,176, DOI: 10.1186/s13068-015-0360-6

69. Shin, H.D. et al. (2014) Comparative engineering of Escherichia coli for cellobiose utilization, hydrolysis versus phosphorolysis. Metab. Eng. 24, 9-17

70. Rutter, C. and Chen, R. (2014) Improved cellobiose utilization in E. coli by including both hydrolysis and phosphorolysis mechanisms. Biotechnol. Lett. 36, 301-307

71. Sekar, R. et al. (2012) Engineering Escherichia coli cells for cellobiose assimilation through a phosphorolytic mechanism. Appl. Environ. Microbiol. 78, 1611-1614

72. Shin, H.D. et al. (2010) Escherichia coli binary culture engineered for direct fermentation of hemicellulose to a biofuel. Appl. Environ. Microbiol. 76, 8150-8159

73. Kim, H. et al. (2014) Analysis of cellodextrin transporters from Neurospora crassa in Saccharomyces cerevisiae for cellobiose fermentation. Appl Microbiol Biotechnol 98, $1087-1094$

74. Bae, Y.H. et al. (2014) Molecular cloning and expression of fungal cellobiose transporters and $\beta$-glucosidases conferring efficient cellobiose fermentation in Sacharomyces cerevisiae. J. Biotechnol 169, 34-41

75. Lane, S. et al. (2015) Development and physiological characterization of cellobioseconsuming Yarrowia lipolytica. Biotechnol. Bioeng. 112, 1012-1022

76. Berlin, A. and Balakshin, M. (2014) Industrial Lignins, Analysis, Properties, and Applications. In Bioenergy Research: Advances and Applications (Gupta, V.K., Tuohy, M., Kubicek, C.P., Saddler, J. and Xu, F., eds), pp 315-336, Elsevier B.V. 
77. Pérez, J. et al. (2002) Biodegradation and biological treatments of cellulose, hemicellulose and lignin, an overview. Int. Microbiol. 5, 53-63.

78. Xu, C. et al. (2014). Lignin depolymerisation strategies, towards valuable chemicals and fuels. Chem. Soc. Rev. 43, 7485-7500.

79. Wang, H. et al. (2013) Recent Development in Chemical Depolymerization of Lignin, A Review. J. Appl. Chem. Article ID 838645, http://dx.doi.org/10.1155/2013/838645

80. Rodrigues Negrão, D. R. et al. (2015) Fragmentation of lignin from organosolv black liquor by white rot fungi. BioResources 10, 1553-1573.

81. Bi, R. et al. (2012) Isolation and identification of microorganisms from soil able ot live on lignin as a carbon source and to produce enzymes which cleave the $\beta-\mathrm{O}-4$ bond in a lignin model compound. Cellulose Chem. Technol. 46, 227-242

82. Christopher, L. P. et al. (2014). Lignin biodegradation with laccase-mediator systems. Frontiers Energy Res. 2. DOI: 10.3389/fenrg.2014.00012

83. Pollegioni, L. et al. (2015) Lignin-degrading enzymes. FEBS Journal 282, 1190-1213.

84. Palma, C. et al. (2016). Production of versatile peroxidase fromPleurotus eryngiiby solid-state fermentation using agricultural residues and evaluation of its catalytic properties. Prep Biochem Biotechnol. 46, 200-207, DOI: 10.1080/10826068.2015.1084513.

85. Bugg, T.D. and Rahmanpour, R. (2015) Enzymatic conversion of lignin into renewable chemicals. Curr Opin Chem Biol. 29, 10-7.

86. Changzhi, Li. et al. (2015) Catalytic Transformation of Lignin for the Production of Chemicals and Fuels. Chem. Rev. 115, 11559-11624.

87. Tian, J.-H. et al. (2014) Occurrence of lignin degradation genotypes and phenotypes among prokaryotes. Appl Microbiol Biotechnol. 98, 9527-9544. 
88. Sainsbury, P.D. et al. (2013) Breaking down lignin to high-value chemicals: The conversion of lignocellulose to vanillin in a gene deletion mutant of Rhodococcus jostii RHA1. ACS Chem Biol 8, 2151-2156

89. Kosa, M., and Ragauskas A.J. (2013) Lignin to lipid bioconversion by oleaginous Rhodococci. Green Chem, 15, 2070-2074

90. Linger, J.G. et al. (2014) Lignin valorization through integrated biological funnelling and chemical catalysis. Proc Natl Acad Sci U S A, 111, 12013-12018

91. Vardon, D.R. et al. (2015) Adipic acid production from lignin. Energy Environ Sci, 8, $617-628$

92. Baker, D.A. and Rials, T.G.(2013) Recent advances in low-cost carbon fiber manufacture from lignin. J Appl Polym Sci, 130, 713-728

93. Yiamsawas, D. et al. (2014) Biodegradable lignin nanocontainers. RSC Adv. 4, $11661-11663$

94. Jeon, J.-W. et al. (2015) Controlling porosity in lignin-derived nanoporous carbon for supercapacitor applications. Chem Sus Chem. 8, 428-432 


\section{Figure Legends:}

Figure1. Deconstruction of lignocellulose by fungal enzymes. The production of extracellular lignin-modifying enzymes that rupture the intact lignin structure initiates the lignocellulose degradation process.

Figure 2. Oxidative and hydrolytic cleavage of crystalline cellulose to monomeric sugars. Amorphogenesis-inducing proteins such as swollenin or expansin-like proteins expedite swelling of cellulose fibers. In doing so, this opens up the plant cell wall and facilitates increased access to the glycosidic linkages within the sugar polymers by cellulases. Lytic polysaccharide monooxygenases (LPMOs) are copper-dependent metalloenzymes that oxidatively split glycosidic bonds on the surface of cellulose matrix by removing hydrogen from $\mathrm{C} 1$ or $\mathrm{C} 4$ of the glucose monomer and inserting oxygen.

Figure 3. A profile of hemicellulase action in lignocellulosic substrate. 


\section{Glossary}

Biomass: a biological material derived from living or dead plants and animals. In the context of biomass for energy, it is often used to mean plants and plants-derived materials.

Biomass feedstock: a renewable biological material of plant and algal origin that can be used directly as a fuel or converted to different forms of fuel and biobased products such as ethanol, butanol, biodiesel, hydrocarbons, lactic acid, succinic acid, propanediol, etc.

Biorefinery: a facility that processes lignocellulosic biomass into different classes or grades of fuels and chemicals.

Carbohydrate Active Enzyme (CAZy): a database that describes enzymes that form and breakdown complex carbohydrates and glycoconjugates, and they typically belong to glycoside hydrolases (GHs), carbohydrate esterases (CEs), polysaccharide lyases (PLs), glycosyltransferases (GTs), and carbohydrate-binding modules (CBMs) of catalytic activities.

Carbohydrate-binding module (CBM): a domain in a carbohydrate-active enzyme that binds to the substrate to bring the catalytic domain of the enzyme into close proximity and prolonged association with the substrate to facilitate and sustain catalysis.

Carbohydrate esterase (CE): a group of enzymes that catalyze the removal of orthoester [O- (ester)] and N-acetyl moieties from carbohydrates.

Energy crops: low-maintenance plants that can be grown on marginal lands, harvested upon maturity, and either combusted to generate heat and electricity or used as a feedstock for the production of liquid fuels.

Expansin: a plant protein with cell-wall loosening activity which may play important roles in cell enlargement and other developmental processes that contributes to cell-wall modification. 
Glycoside hydrolase (GH): an enzyme that catalyzes the hydrolysis of glycosidic linkage of glycosides to form sugar hemiacetal or hemiketal and the corresponding free aglycon.

LDMICs (lignocellulose-derived microbial inhibitory compounds): are microbial inhibitory compounds such as furfural, hydroxymethylfurfural (HMF), p-coumaric acid, glucuronic acid, syringaldehyde, ferulic acid, syringic acid, and 4-hydroxybenzaldehyde that are typically generated during dilute acid pretreatment and hydrolysis of lignocellulosic biomass.

Lignin: a non-carbohydrate component of lignocellulosic biomass is a heterogeneous polymer of three alcohol monomer, p-coumaryl alcohol, coniferyl alcohol, and sinapyl alcohol.

Lignocellulose: a generic term used to describe plant materials whose major components are lignin, cellulose, and hemicelluloses.

Lytic polysaccharide monooxygenases (LPMOs): a class of enzymes that oxidatively cleave recalcitrant polysaccharides including cellulose.

Polysaccharide lyase: a group of enzymes that catalyze non-hydrolytic breakdown of uronic acid-containing polysaccharides via a $\beta$-elimination mechanism to form unsaturated hexenuronic acid residues and new reducing ends.

Swollenin: a non-hydrolytic protein that plays important role in amorphogenesis of cellulose during which the cellulose structure is non-hydrolytically disrupted or opened up thereby enhancing its accessibility by hydrolytic enzymes.

Synergy: a phenomenon in which combined activities of two or more enzymes exceed the arithmetic sum of the activity of each enzyme. 


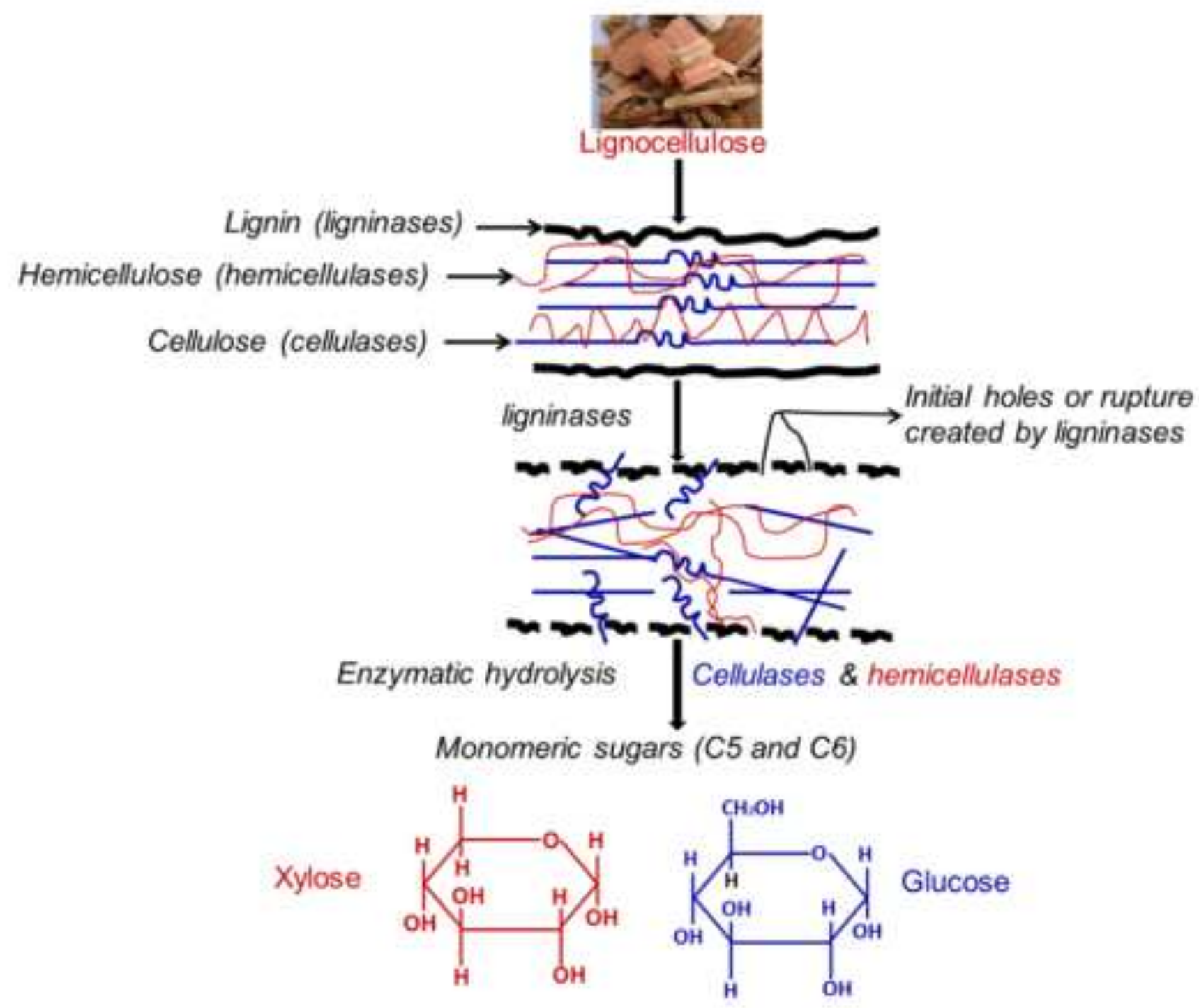




\section{Crystalline cellulose}

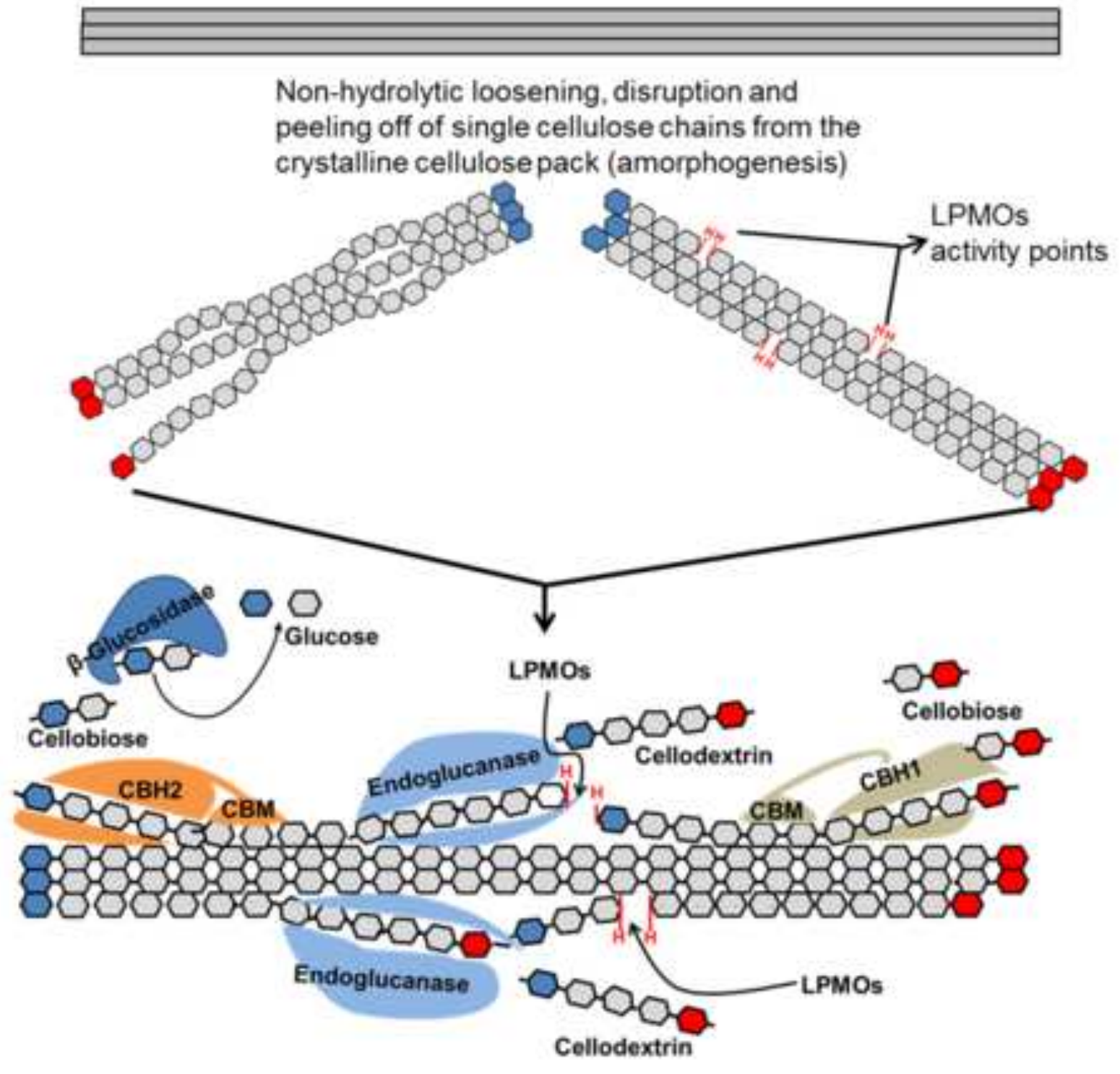

peeling off of single cellulose chains from the

crystalline cellulose pack (amorphogenesis) 
Lignocellulosic residues

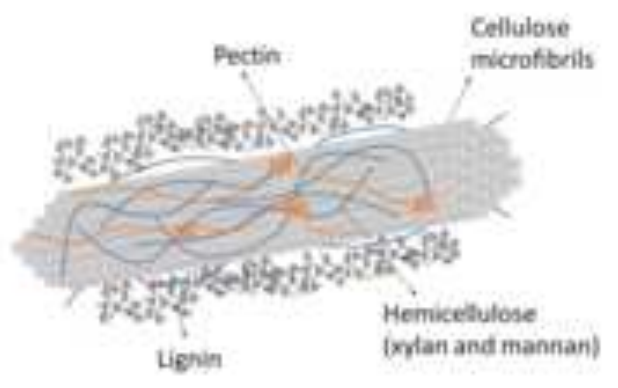

Pretreatment

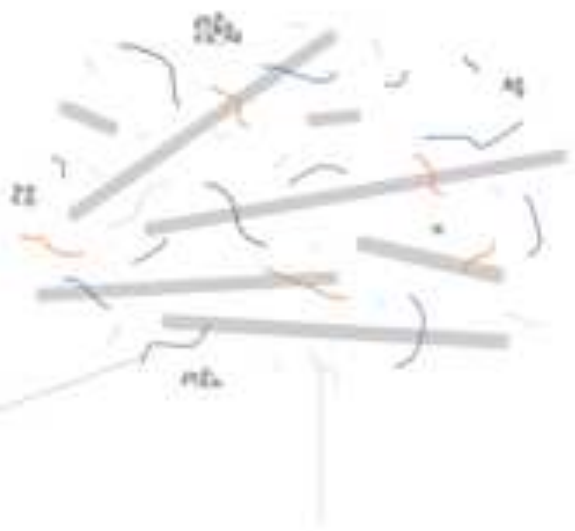

Xylan-degrading enrymes

Mannan-degrading enzymes
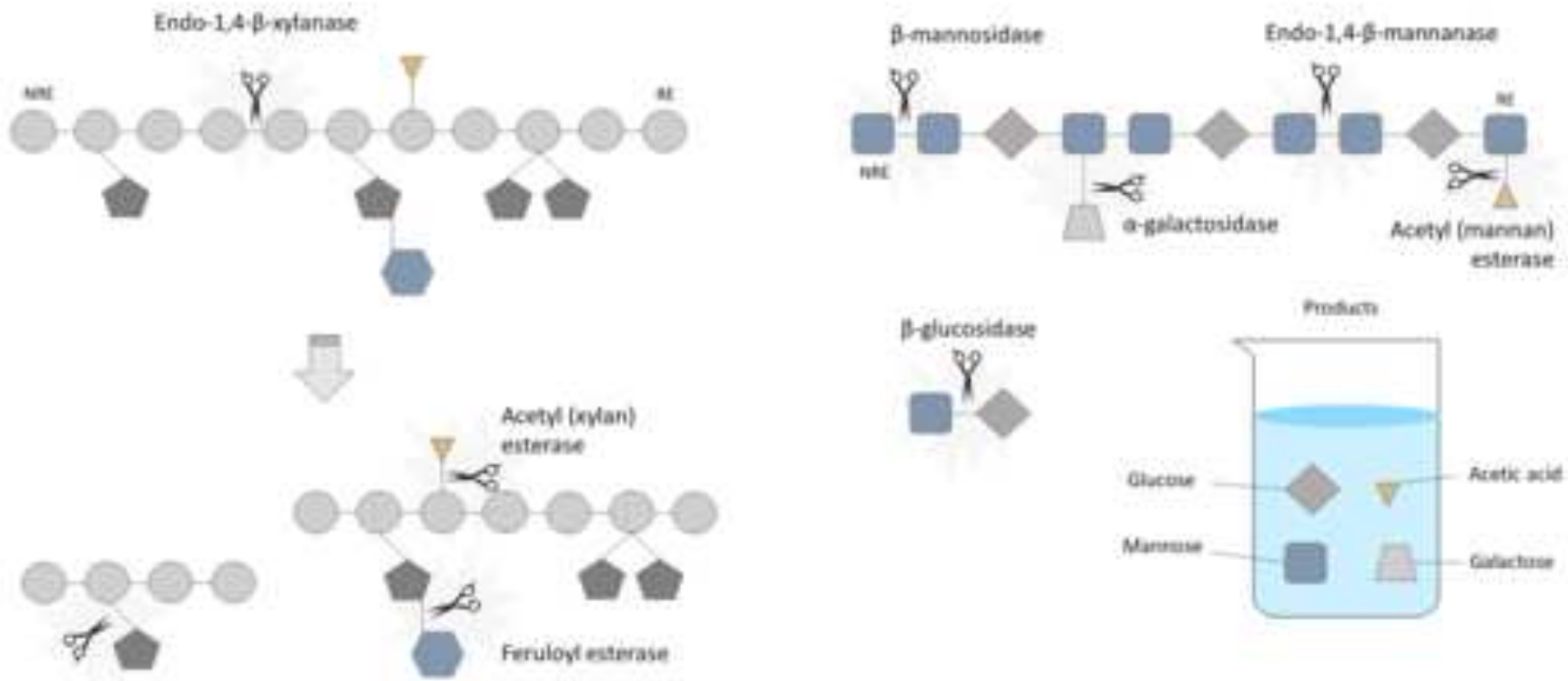

B-evicosidase

a-arabinefuranosidate
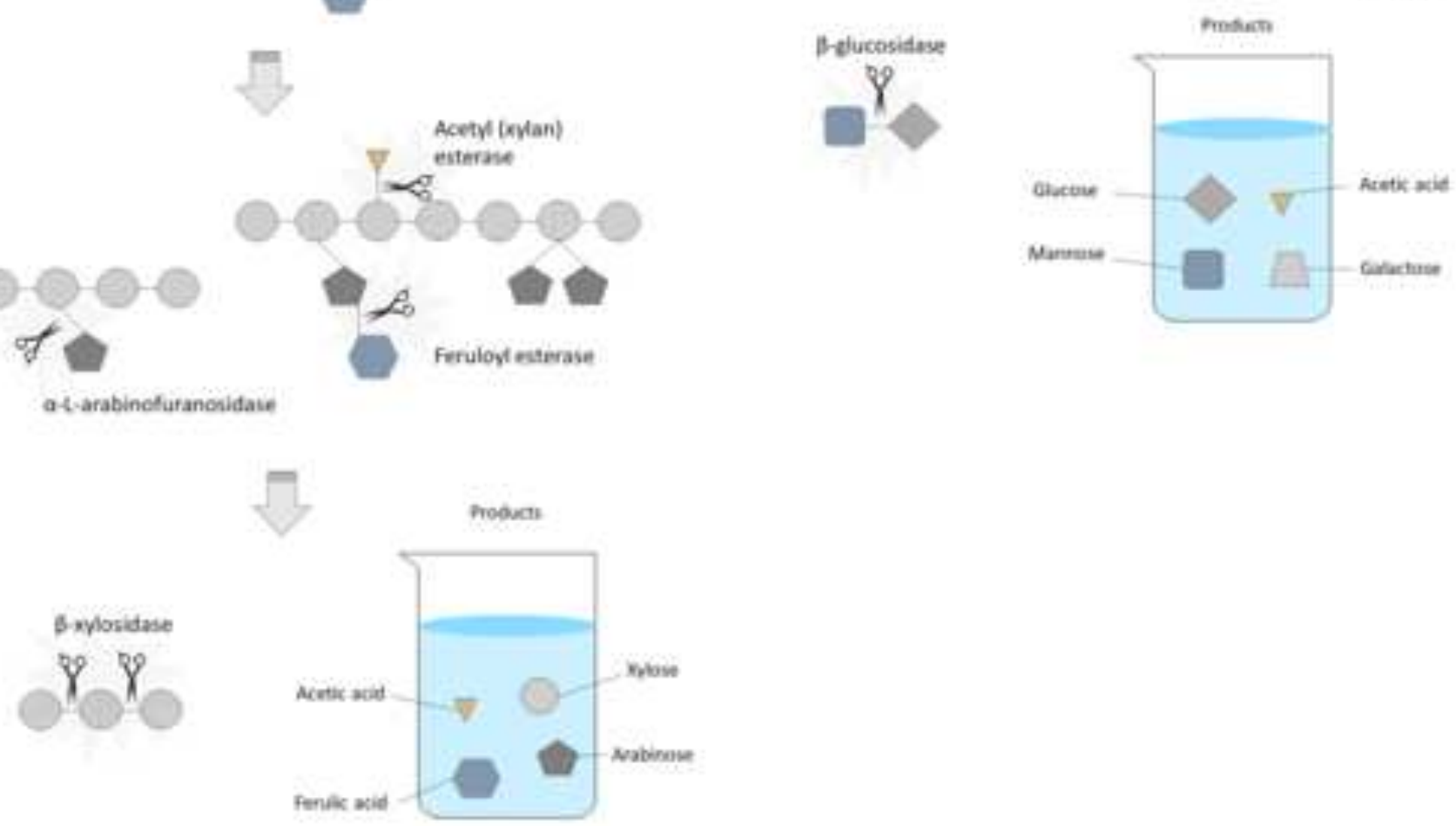
Table 1. Biorefinery products that can be produced from lignocellulose hydrolysates

\begin{tabular}{|c|c|c|}
\hline Product & Producing 0 & ganism \\
\hline & Prokaryotes & Eukaryotes \\
\hline (2R,3R)-2,3-Butanediol & Enterobacter cloacae & \\
\hline 1,2,4-Butanetriol & Escherichia coli & \\
\hline 1,4-Butanediol & E. coli & \\
\hline 1,5-Diaminopentane & Corynebacterium glutamicum & \\
\hline 2,3-Butanediol & $\begin{array}{l}\text { Klebsilla oxytoca, Paenibacillus } \\
\text { polymyxa, E. coli }\end{array}$ & \\
\hline $\begin{array}{l}\text { ABE } \\
\text { (acetone/butanol/ethanol) }\end{array}$ & $\begin{array}{l}\text { Clostridium acetobutylicum, } C \text {. } \\
\text { beijerinckii }\end{array}$ & \\
\hline Acetoin & E. coli, Bacillus subtilis & \\
\hline a-Ketoglutarate derivatives & C. glutamicum & \\
\hline Butanol & $\begin{array}{l}\text { C.acetobutylicum, } C . \\
\text { beijerinckii, C. tyrobutyricum }\end{array}$ & \\
\hline Caffeic acid & E.coli & \\
\hline Cinnamic acid & E. coli & \\
\hline d-Lactate & E. coli, Lactobacillus plantarum & Kluyveromyces lactis \\
\hline Ethanol & $\begin{array}{l}\text { Zymomonas mobilis, E. coli, C. } \\
\text { glutamicum }\end{array}$ & $\begin{array}{l}\text { Saccharomyces cerevisiae, } \\
\text { Candida utilis, } \\
\text { Kluyveromyces } \\
\text { marxianum, Pichia stipidis }\end{array}$ \\
\hline
\end{tabular}




\begin{tabular}{|l|l|l|}
\hline Ethylene glycol & E. coli, L. plantarum & \\
\hline Glycolic acid & & S. cerevisiae, K. lactis \\
\hline Isobutanol & Ralstonia eutropha & \\
\hline $\begin{array}{l}\text { PHB (poly-beta- } \\
\text { hydroxybutyrate) }\end{array}$ & E. coli & \\
\hline p-Hydroxycinnamic acid & E. coli & \\
\hline $\begin{array}{l}\text { Poly(lactate-co-3- } \\
\text { hydroxybutyrate) (P(LA-co- } \\
\text { 3B)) }\end{array}$ & E. coli & S. cerevisiae \\
\hline $\begin{array}{l}\text { Poly-3-d-hydroxybutyrate } \\
\text { (PHB) }\end{array}$ & E. coli, C. glutamicum & S. cerevisiae, \\
\hline Succinate & E. coli & \\
\hline Xylitol & Xebaryomyces hansenii \\
\hline Xylonic acid & & \\
\hline
\end{tabular}

* Data extracted from Chen and Dou, 2015 [54] with modification 